16. DANDAPAT, M.C.; MOHAPATRO, S.K.; PATRO, S.K.: "Elephantiasis of the penis and scrotum. A review of 350 cases". American Journal of Surgery, 149: 686, 1985.

17. KONETY, B.R. y cols.: "Scrotal elephantiasis associated with hidradenitis suppurativa". Plastic and Reconstructive Surgery, 97: 1243, 1996.

*18. MALLOY, T.R.; WEIN, A.J.; GROSS, P.: "Scrotal and penile lymphedema: surgical considerations and management". J. Urol., 130: 263, 1983.

19. ROSS, J.H. y cols.: "Primary lymphedema of the genitalia in children and adolescents". J. Urol., 160: 1485, 1998.

20. Mc BRIEN, M.P.: "Of the testes". Arch. Surg., 104: $820,1972$.

Casos Clínicos

Arch. Esp. Urol., 60, 6 (692-694), 2007

\section{CISTITIS POLIPOIDE ASOCIADA A CISTITIS QUÍSTICA GLANDULAR.}

Francisco Javier Torres Gómez', Francisco Javier Torres Olivera ${ }^{2}$ y Amelia Torres Gómez².

Servicio de Anatomía Patológica'. Hospital Punta de Europa. Algeciras. Cádiz. y Departamento de Anatomía Patológica? Hospital Universitario Virgen Macarena. Sevilla. España.
Resumen.- OBJETIVO: La cistitis polipoide y la metaplasia intestinal son dos lesiones de sobra conocidas a nivel vesical

MÉTODOS: Presentamos el caso de un paciente en cuya vejiga se identificaron ambas lesiones de modo sincrónico.

RESULTADO: Si bien ambas lesiones son no neoplásicas, existen evidencias que apuestan por una posible degeneración del epitelio metaplásico hacia adenocarcinoma.

CONCLUSIONES: El diagnóstico de ambas lesiones es histológico no existiendo pruebas clínicas ni estudios de imagen que permitan identificar la verdadera naturaleza de dichas lesiones.

Palabras clave: Cistitis. Polipoide. Metaplasia intestinal. Glandular.

Summary.- OBJECTIVE: Polypoid cystitis and intestinal metaplasia are well-known lesions of the bladder.

METHODS: We report the case of one patient with both lesions identified synchronically in the bladder.

RESULTS: Although these lesions are not neoplastic, there are evidences supporting a possible degeneration of the metaplastic epithelium to adenocarcinoma.

CONCLUSIONS: The diagnosis of both lesions is histological and there are not clinical tests or image studies that could enable identification of the real nature of these lesions.

Keywords: Cystitis. Polypoid. Intestinal metaplasia. Glandular.

\section{INTRODUCCIÓN}

Tanto la cistitis polipoide como la cistitis glandular son dos lesiones pseudotumorales a nivel vesical cuya manifestación sincrónica es infrecuente. Si bien ninguna de ellas tiene un potencial maligno "per se", se debe realizar un diagnóstico diferencial con lesiones que sí lo poseen.

\section{Caso clínico}

Paciente varón de 79 años con síndrome prostático cuyo estudio clínico reveló una próstata de gran tamaño, nodular y de consistencia firme con lo cual se realizó una resección transuretral (RTU). El material resecado se envió para estudio histopatológico confirmándose el 


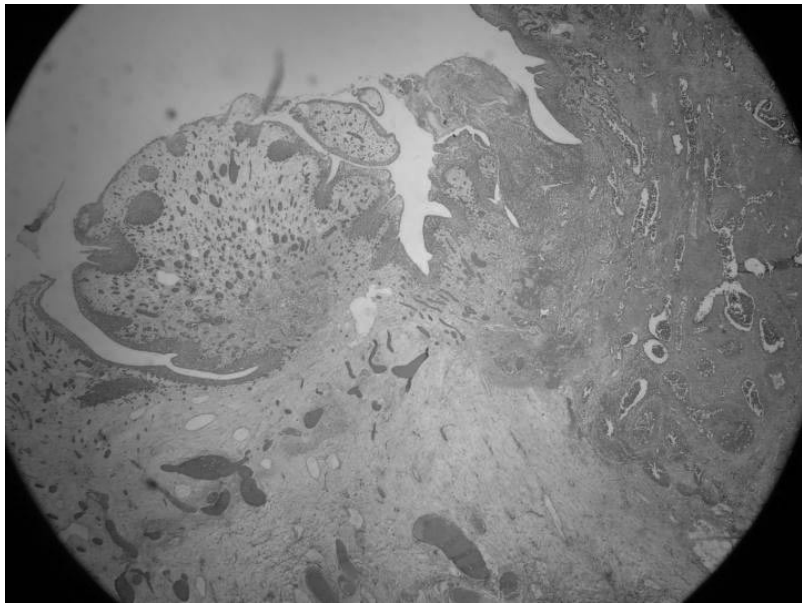

FIGURA 1. Cistitis polipoide. Visión panorámica 5x.

diagnóstico de hiperplasia nodular. Al mismo tiempo se realizó una cistoscopia en la que se observó una lesión excrecente en el fondo vesical, con aspecto tumoral. Esta lesión fue asimismo ampliamente extirpada mediante RTU.

El estudio microscópico de la misma permitió observar una mucosa edematosa con gran congestión vascular que emitía prolongaciones sesiles a modo de pólipos hacia la luz, poseyendo éstas un tamaño heterogéneo y estando tapizadas por un epitelio de tipo transicional ligeramente hiperplásico (cistitis polipoide) (Figuras $1,2,3$ ). Llamaba especialmente la atención la presencia de células caliciformes (metaplasia intestinal) tanto a nivel del epitelio de superficie como a nivel de

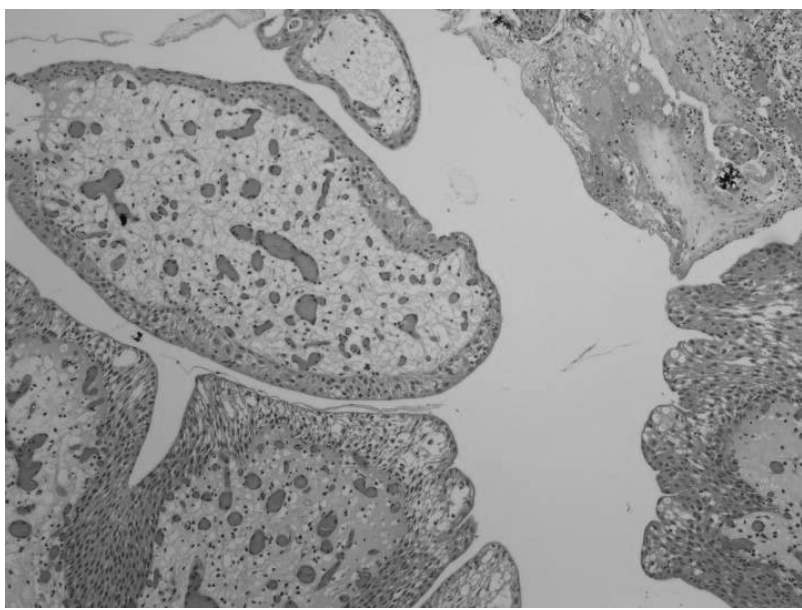

FIGURA 3. Cistitis polipoide. Detalle de los pólipos 20x.

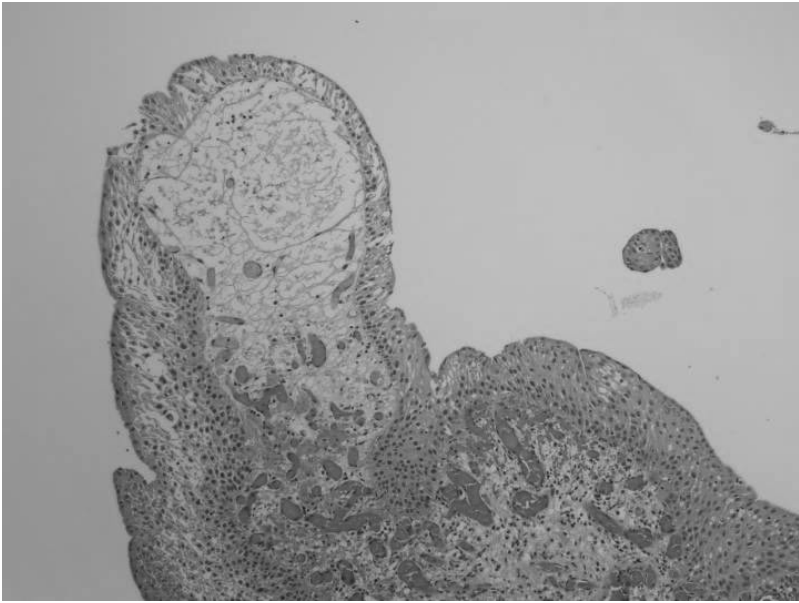

FIGURA 2. Cistitis polipoide. Detalle de los pólipos 10x.

glándulas infraepiteliales distribuidas al azar a nivel del corion (cistitis quística glandular) (Figuras 4 y 5). Con tales hallazgos se emitió el diagnóstico de cistitis polipoide y de cistitis quística glandular (presentación sincrónica).

\section{DISCUSIÓN}

La cistitis polipoide es una lesión fumoral benigna de carácter reactivo que puede simular una auténtica neoplasia tanto en el examen clínico como en los estudios por imagen por lo cual será un minucioso estudio histológico el que determine la verdadera naturaleza de la lesión. Si bien cualquier localización es posible a la

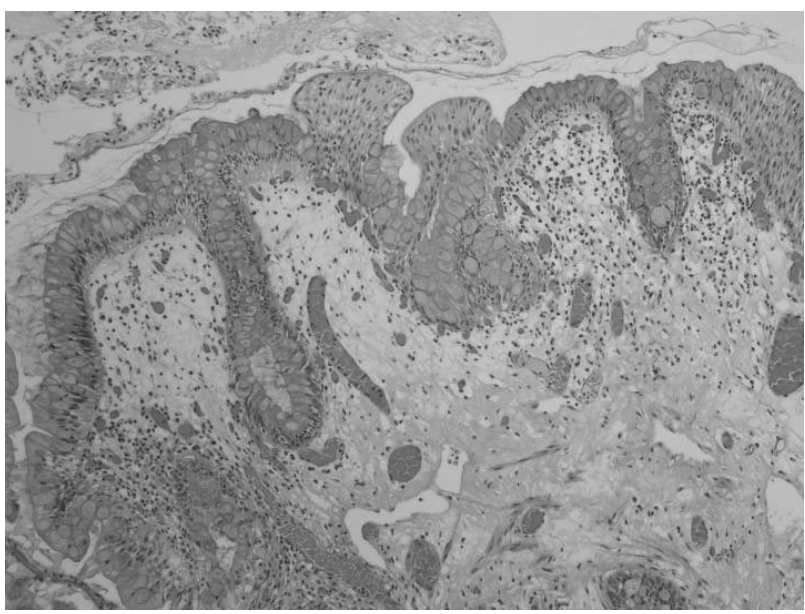

FIGURA 4. Metaplasia intestinal del epitelio de superficie $10 x$ 
largo de la mucosa vesical, existe una predilección por la pared posterior o por la cúpula vesical. Tradicionalmente se ha subclasificado la cistitis polipoide en dos grupos atendiendo a la morfología de las proyecciones polipoides; así, en la cistitis polipoide bullosa éstas serán anchas y de bordes romos mientras que en la cistitis polipoide papilar se observarán proyecciones delgadas y filiformes. Esta clasificación "como es lógico suponer" no tiene ningún impacto clínico- pronóstico si bien es necesario hacer hincapié en la distinción, en la mayor parte de las ocasiones fácil, del tipo papilar con el verdadero carcinoma de células transicionales papilar, auténtica neoplasia (6).

Existe una alta relación de la cistitis polipoide con la instrumentalización-cateterización vesical $(75 \%$ de los casos de sondaje vesical mantenido) (1) alcanzándose su mayor incidencia a los tres meses del sondaje y desapareciendo al retirar la sonda; asociaciones con radiación o neoplasias malignas han sido descritas En el $25 \%$ de los casos no se identifican antecedentes de interés.

Es importante destacar el curso inocuo de esta patología, con una evolución favorable en gran parte de los casos si bien se han documentado casos en que debido a la obstrucción de los uréteres se han desarrollado hidronefrosis e insuficiencia renal (5). También se han documentado asociaciones con displasia epitelial hasta en un $6 \%$ de los casos si bien todos los indicios apuntan a una aparición sincrónica más que a una evolución.

El caso que presentamos muestra la asociación de cistitis polipoide con cistitis quística glandular, asociación infrecuente. La historia natural del desarrollo de metaplasia intestinal a nivel de la mucosa vesical parece re-

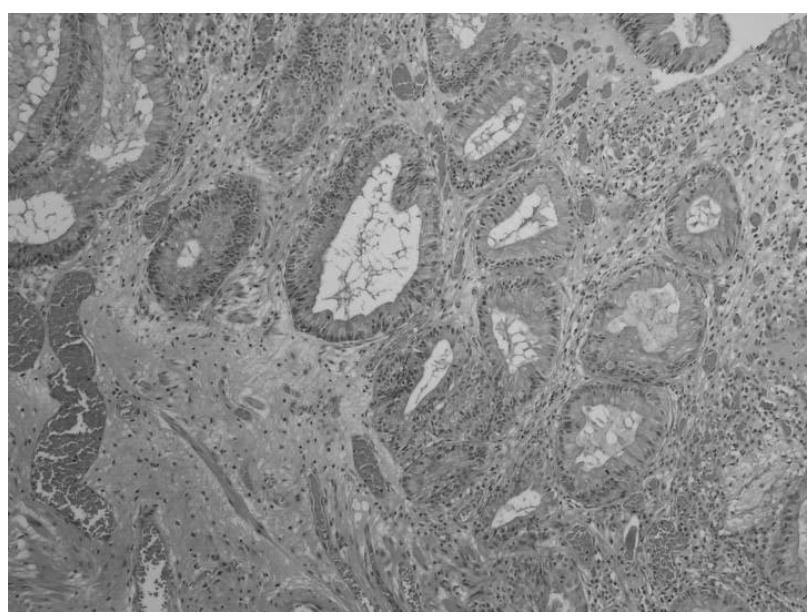

FIGURA 5. Metaplasia intestinal. Imágenes glandulares en lámina propia 10x. lacionarse con cambios inflamatorios crónicos, infección u otras causas irritativas como pueden ser el prolapso o la exostrofia, lesiones ambas íntimamente asociadas a este cambio $(3,8)$.

Se trata de una patología sin expresión clínica; no obstante aquellos casos con amplios focos de metaplasia intestinal han sido relacionados con potencial neoplásico (adenocarcinoma vesical) (2)por lo que se trata de pacientes que deben someterse a un seguimiento para evitar este último supuesto (la cistitis glandular se encuentra presente en $14-67 \%$ de los casos de adenocarcinoma vesical).

Cuando se asocian cistitis polipoide y metaplasia intestinal es lógico suponer que el pronóstico se relacionará con la degeneración o no de los focos metaplásicos si bien no existen trabajos que aborden esta peculiar asociación. Lo que sí podemos afirmar es que nuestro paciente lleva dos años sin sintomatología ni evidencias citológicas e histológicas de neoplasia.

En aquellos casos que cursan con obstrucción ureteral o aquellos que reduzcan drásticamente la capacidad vesical será necesario llevar a cabo procedimientos quirúrgicos más complicados con el fin de evitar el desarrollo de hidronefrosis y consecuentemente de la temida insuficiencia renal (7).

\section{BIBLIOGRAFÍA Y LECTURAS \\ RECOMENDADAS (*lectura de interés $y^{* *}$ lectura fundamental)}

*1. EKELUND, P.; JOHANSSON, S.: "Polypoid cystitis. A catheter associated lesion of the human bladder". Acta Pathol. Microbiol. Scand., 87: 179, 1979.

2. ABENOZA, P.; MANIVEL, C.; FRALEY, E.E.: "Primary adenocarcinoma of urinary bladder. Clinico pathologic study of 16 cases". Urology, 29: 9, 1987.

**3. BULLOCK, P.S.; THONI, D.E.; MURPHY, W.M.: "The significance of colonic mucosa (intestinal metaplasia) involving the urinary tract". Cancer, 59: 2086, 1987.

4. YOUNG, R.H.: "Papillary and polypoid cystitis. A report of eight cases". Am. J. Surg. Pathol., 12: 542, 1988.

5. VALERO PUERTA, J.A.; MEDINA PEREZ, M.; VALPUESTA FERNANDEZ, I. y cols.: "Bilateral hydronephrosis with papillary polypoid cystitis". Arch. Esp. Urol., 52: 396, 1999.

6. BORDA, A.; PETRUCCI, M.D.; BERGER, N.: "Miscellaneous benign lesions of the bladder and the urinary tract". Ann. Pathol., 24: 18, 2004.

7. BLACK, P.C.; LANGE, P.H.: "Cystoprostatectomy and neobladder construction for florid cystitis glandularis". Urology, 65: 174, 2005.

8. NOVAK, T.E.; LAKSHMANAN, Y. y cols.: "Polyps in the exostrophic bladder. A cause for cancer?". J. Urol., 174: 1522, 2005. 